\title{
Oxygen uptake versus exercise intensity: a new concept in assessing cardiovascular exercise function in patients with congenital heart disease
}

\author{
T Reybrouck, L Mertens, S Brusselle, M Weymans, B Eyskens, J Defoor, M Gewillig
}

\begin{abstract}
Objective-To assess the relation between exercise intensity and oxygen uptake during graded exercise in paediatric patients who underwent surgical repair of congenital heart disease, and to compare it with conventional measures of aerobic exercise function.

Design-Cross sectional study. Exercise testing was performed on a treadmill and gas exchange was measured on a breath by breath basis.

Patients-29 patients who underwent an atrial switch operation for transposition of the great arteries (TGA) (mean (SD) age at testing 10.3 (2.5) years) and 30 patients who underwent total repair of tetralogy of Fallot (TF) (age 12.1 (3.3) years) performed graded exercise testing. Exercise responses were compared with data obtained in 24 normal controls (age 11.4 (2.6) years).

Results-The slope of oxygen uptake versus exercise intensity averaged 1.50 $(0.64) \mathrm{ml} \mathrm{O}_{2} / \mathrm{min}^{2} / \mathrm{kg}$ in the patients with TGA and $1.68(0.75) \mathrm{ml} \mathrm{O} / \mathrm{min}^{2} / \mathrm{kg}$ after TF repair, both lower $(\mathrm{p}<0.005)$ than in normal controls $\left(2.42(0.68) \mathrm{ml} \mathrm{O}_{2} / \mathrm{min}^{2} / \mathrm{kg}\right)$. The lower slope of oxygen uptake was correlated with a subnormal value for ventilatory anaerobic threshold, which averaged 78.0 (13.3)\% of normal in TGA and $85.1(10.6) \%$ in TF. This was associated with a steeper slope $(\mathrm{p}=0.001)$ of carbon dioxide output versus oxygen uptake above the ventilatory anaerobic threshold in TGA $(1.26(0.20))$ and TF $(1.20(0.18))$ compared with the normal controls $(1.05(0.13)$ ), and also a steeper slope of ventilation versus carbon dioxide in TGA (47.0 (15.4)) and TF (41.5 (13.7)) than in the controls (30.3 (8.5)).

Conclusions-Calculation of the steepness of the slope of oxygen uptake versus exercise intensity is a valid measurement of oxygen flow to the exercising tissues, which may be limited in congenital heart disease.

(Heart 2000;84:46-52)
\end{abstract}

Keywords: congenital heart disease; exercise testing; oxygen uptake; oxygen uptake kinetics

There are various different ways of assessing cardiorespiratory exercise capacity under clinical conditions. Traditionally, methods that assess maximum exercise capacity have been used, such as exercise time or maximum oxygen uptake. These variables are influenced by the patient's motivation when performing a maximum exercise test or by noncardiorespiratory symptoms that lead to premature termination of the test. Maximum exercise capacity is also of little relevance in daily life activities. In order to circumvent these limitations, submaximal indices of cardiorespiratory function have been proposed, such as the ventilatory anaerobic threshold, ${ }^{1}$ though concerns have been raised about the reliability of the techniques used to measure this threshold. ${ }^{2-4}$ Another method involves determining the slope of carbon dioxide output against oxygen uptake, which is considered to be a useful and reproducible index of cardiovascular exercise function in patients with chronic heart failure ${ }^{5}$ and congenital heart disease. ${ }^{6}$ This method, however, also depends on determining the anaerobic threshold.

A further approach to assessing aerobic exercise function is to analyse the slope of the relation between exercise intensity and oxygen uptake during graded exercise testing. In general, the relation between work rate and oxygen uptake can be described by a linear function, both for steady state and non-steady state exercise. ${ }^{78}$ In patients with chronic heart failure, the increase in oxygen uptake cannot meet the demand at higher levels of exercise, ${ }^{9}$ and there is a rise in carbon dioxide production caused by a switch to an increased reliance on glycolysis.

Limited information is available about the slope of oxygen uptake versus exercise intensity in patients with congenital heart disease. ${ }^{10}$ Furthermore, most studies on the relation between oxygen uptake and work rate in both normal controls and cardiac patients have been performed using bicycle ergometry, ${ }^{7-11}$ which can be difficult in young patients. ${ }^{10}$

We have now used graded treadmill exercise - the most commonly used technique in paediatric exercise testing - to analyse the relation between exercise intensity and oxygen uptake during non-steady state exercise in children and adolescents with surgically corrected congenital heart disease. We hypothesised that the slope of the increase in oxygen uptake versus exercise intensity would be lower in patients with congenital heart disease, and that it would correlate with the degree of exercise limitation as assessed by conventional exercise tests.

\section{Methods}

SUBJECTS

Exercise tests were performed in three groups of subjects (two patient groups and one normal control group). 
The first group consisted of 29 patients (19 boys and 10 girls), who underwent cardiopulmonary exercise testing 9.9 years after an atrial switch operation for transposition of the great arteries (TGA). The Senning operation was performed in 27 subjects and the Mustard procedure in two. Age at surgery varied from 1 to 19 months, mean (SD) 5.4 (4.8) months, and age at exercise testing varied from 5.7 to 14.7 years, mean 10.3 (2.5) years. All these patients were in sinus rhythm at the time of exercise testing. Echocardiographic evaluation at that time showed patent venous pathways in all but one patient.

A second group of 30 patients (18 boys and 12 girls) was studied 8.3 (4.4) years after total surgical repair of tetralogy of Fallot (TF). Age at surgery varied from 0.75 to 11.5 years (mean 3.7 (2.8) years), and age at exercise testing varied from 5.5 to 16.7 years (mean 12.1 (3.3) years).

The values for the slope of oxygen uptake versus exercise intensity in the two patient groups were compared with a third group of 24 normal controls of comparable age (7.2 to 17.7 years, mean 11.4 (2.6) years). These normal controls were children and adolescents who were referred to our paediatric cardiology outpatient clinic for a heart murmur (or functional complaints), and in whom a definite diagnosis of a functional heart murmur in a morphologically and functionally normal heart was made. The diagnosis was based on clinical findings, ECG, and echocardiography.

All patients with TF repair and atrial switch operation for TGA were asymptomatic at the time of exercise testing. On the ECG, right bundle branch block was found in all patients after TF repair. Body weight was not significantly different $(p>0.05)$ among the three groups of subjects. It averaged $33.9(11.2) \mathrm{kg}$ in patients with TGA, 41.8 (16) $\mathrm{kg}$ in patients after total repair for $\mathrm{TF}$, and $37.5(10.7) \mathrm{kg}$ in the normal controls. When the data for height and weight were expressed as centiles and were compared with a standard growth curve, ${ }^{12}$ one of the 30 patients after TF repair was below the third centile for height and weight. In the group of patients with atrial switch for TGA, one patient was also below the third centile for weight.

Reproducibility studies were performed in 10 normal subjects, varying in age from 8.7 to 22.7 years (mean 17.7 (6.1) years). These subjects underwent two exercise tests within one day.

Informed consent was obtained from the parents or the patients after the nature of the test procedure had been fully explained. The clinical investigations were approved by the local medical ethics committee.

EXERCISE TESTING PROCEDURE

The methods for exercise testing have been fully described previously. ${ }^{6}{ }^{13}$ Briefly, exercise testing was performed on a treadmill, and the speed was set at $4.8 \mathrm{~km} / \mathrm{h}$ for children less than 6 years of age and at $5.6 \mathrm{~km} / \mathrm{h}$ for children six years of age or older. This study was intended to assess exercise performance using submaxi- mal exercise. The inclination of the treadmill was increased by $2 \%$ every minute until the submaximal target heart rate of 170 beats/min was reached. A target heart rate of 170 beats/min was chosen, because this is a conventional limit in cardiovascular exercise testing that has often been used in the past; it corresponds to the highest heart rate of submaximal steady state work and has been shown to correlate with maximum oxygen uptake. ${ }^{1014}$ The oxygen uptake at a heart rate of 170 beats/min was determined.

Gas exchange was measured on a breath by breath basis by a computerised system using a mass spectrometer (Marquette MGA 1100; Milwaukee, USA). ${ }^{13}{ }^{15}$

The adequacy of the oxygen transport system was assessed by calculating the steepness of the slope of oxygen uptake versus exercise intensity during the graded treadmill exercise, using linear regression analysis. In this analysis, exercise intensity was estimated from exercise time (expressed in minutes or fractions of minutes), as all patients performed the same standardised graded exercise test. Therefore the slope of the regression of oxygen uptake (determined as $\mathrm{ml} \mathrm{O}_{2} / \mathrm{min} / \mathrm{kg}$ ) versus exercise intensity (time in minutes) is expressed as $\mathrm{ml} \mathrm{O}_{2} / \mathrm{min}^{2} / \mathrm{kg}$. This regression was calculated on all breaths, averaged every 10 seconds, using the moving average principle, in order to reduce the variability and disturbing effect of noise. The data of the first 60 seconds of exercise were omitted because it is known that the breathing pattern is very irregular at the onset of exercise. ${ }^{68} \mathrm{~A}$ typical example is shown in fig 1.

To account for the influence of body weight, which determines the size of the work incre-

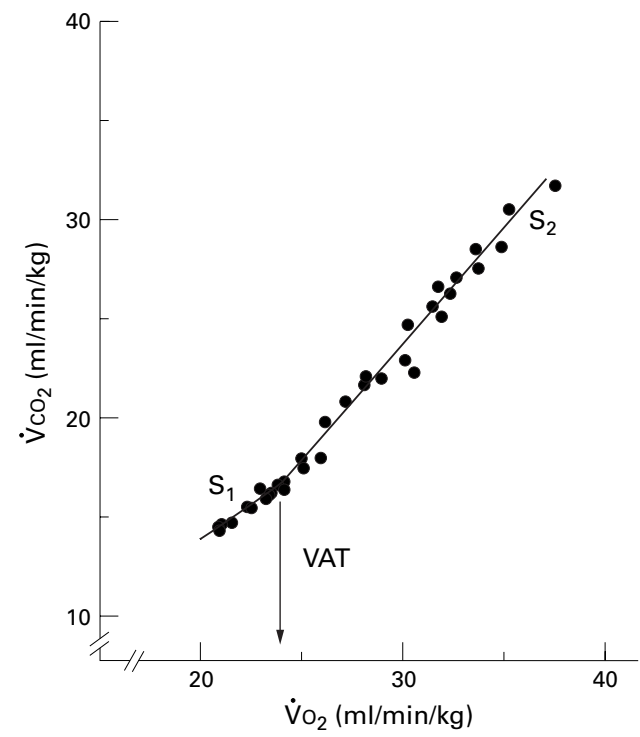

Figure 1 Typical example of the slope of carbon dioxide versus oxygen uptake during graded treadmill exercise in a 14 year old boy with atrial switch operation for transposition of the great arteries. The data represent average values for 10 second intervals. $S 1(=0.79)$ is the slope of carbon dioxide versus oxygen uptake below the ventilatory anaerobic threshold, and $S 2(=1.23)$ is the slope of carbon dioxide versus oxygen uptake above the ventilatory threshold up to a heart rate of 170 beats/min. $\dot{V} \mathrm{CO}_{2}=$ carbon dioxide output, expressed as $\mathrm{ml} / \mathrm{min} / \mathrm{kg}$; $\dot{V}_{\mathrm{O}_{2}}=$ oxygen uptake, expressed as $\mathrm{ml} / \mathrm{min} / \mathrm{kg}$. 
ments on a treadmill, an estimate of the external work for a fixed inclination $(10 \%)$ was calculated using standard equations. ${ }^{16}$

Cardiorespiratory exercise function was further assessed by determining the ventilatory anaerobic threshold. This was defined using the $\mathrm{V}$ slope method and calculated from a computer algorithm. ${ }^{17}$ Data for ventilatory anaerobic threshold were expressed in $\mathrm{ml} / \mathrm{min} /$ $\mathrm{kg}$. As the values for ventilatory anaerobic threshold are to a large extent influenced by age and sex in children of varying age, the results from the patients were also expressed as a percentage of the normal mean value obtained in healthy children of comparable age and sex, extracted from a large pool of 237 normal children. ${ }^{18}$

The oxygen uptake versus exercise intensity slope was also compared with two other recently developed indices used for assessing cardiorespiratory exercise performance. First, we determined the steepness of the slope of carbon dioxide output versus oxygen uptake above the ventilatory anaerobic threshold ${ }^{5619}$; this slope was calculated between the ventilatory anaerobic threshold and the exercise intensity at which a heart rate of 170 beats/min was reached (slope 2). Second, we assessed the slope of ventilation versus carbon dioxide output for all exercise levels, excluding the first minute because of hyperventilation. This was used as an index of ventilatory efficiency. ${ }^{20}$ The respiratory gas exchange ratio $(\mathrm{R})$ was calculated as the ratio of carbon dioxide output to oxygen uptake.

\section{STATISTICAL ANALYSIS}

Differences between the mean values were calculated by univariate analysis of variance. Post hoc analysis of differences between the subgroups was performed by the Bonferroni method. For regression analysis, a linear regression technique was applied, and for correlation analysis we used the Pearson correlation. Reproducibility was determined by calculating the coefficient of repeatability according to Bland and Altman ${ }^{21}$; this was defined as two times the standard deviation of the differences. In addition, the coefficient of variation was defined as 100 times the standard deviation of differences divided by the mean, and the percentage difference was calculated as 100 times the absolute difference between two tests divided by the mean value of both measurements. Significance levels were set at $p<0.05$.

\section{Results}

DETERMINING THE SLOPE OF OXYGEN UPTAKE VERSUS EXERCISE INTENSITY AND RELATED VARIABLES

We were able to calculate the slope of oxygen uptake versus exercise intensity in all subjects. A target heart rate of 170 beats $/ \mathrm{min}$ was reached in all the normal controls. In two of the patients with TF repair a target heart rate of 170 beats/min could not be reached because of exhaustion. In these patients, maximum heart rates were 150 and 153 beats $/ \mathrm{min}$. In three patients with TGA it was also not possible to achieve the target heart rate of 170 beats $/ \mathrm{min}$, because of exhaustion and dyspnoea in two and the development of second degree atrioventricular block in one. In these patients the maximum heart rates were 150 and 167 beats/ min in the first two and 138 beats/min in the patient with atrioventricular block. In these subjects, the slope of oxygen uptake versus exercise intensity was calculated using the maximum exercise heart rate achieved.

The oxygen uptake at a heart rate of 170 beats $/ \mathrm{min}$ averaged $29.9(7.7) \mathrm{ml} / \mathrm{min} / \mathrm{kg}$ in the patients with TGA and $30.4(6.3) \mathrm{ml} /$ $\mathrm{min} / \mathrm{kg}$ in the patients with $\mathrm{TF}$ repair, versus $36.5(5.3) \mathrm{ml} / \mathrm{min} / \mathrm{kg}$ in the normal controls ( $p<0.001$; patients $v$ controls). The relation between heart rate and oxygen uptake in both the patient groups and the normal controls is shown in fig 2 . The lowest value for oxygen uptake at a heart rate of 170 beats/min was reached in patients with TGA. For a further assessment of the relative exercise intensity performed at a heart rate of 170 beats/min, we determined the $\mathrm{R}$ value at that heart rate, and also the level of exercise (per cent inclination of the treadmill) at which an $R$ value of 1.0 was achieved. $\mathrm{R}$ at a heart rate 170 beats/min averaged $0.97(0.08)$ in the patients with TGA, $0.96(0.07)$ in the patients with $\mathrm{TF}$ repair, and $0.90(0.07)$ in the normal controls $(\mathrm{p}<0.005$; patients $v$ controls). The exercise level at which $\mathrm{R}$ reached 1.0 averaged $7.2(2.7) \%$ in the patients with TGA, which was less $(p<0.001)$ than the values obtained in the patients with $\mathrm{TF}$ repair $(12.2(3.1) \%)$ and in the normal controls (12.9 (3.9)\%).

The ventilatory anaerobic threshold could not be determined in 13 of the 59 patients $(22 \%)$ (six in the TGA group and seven in the TF group). This was a prerequisite for subsequent analysis of the slopes of carbon dioxide output versus oxygen uptake above the ventilatory anaerobic threshold. In the normal controls we were able to determine the ventilatory anaerobic threshold in all cases.

A reproducibility study was performed in 10 normal subjects. These performed two exercise tests, separated by a minimum of 24 hours. The coefficient of repeatability was $0.33 \mathrm{ml} \mathrm{O}_{2} /$ $\mathrm{min} / \mathrm{kg}$. The coefficient of variation was $3.6 \%$ and the percentage difference amounted to 5.5 $(4.4) \%$.

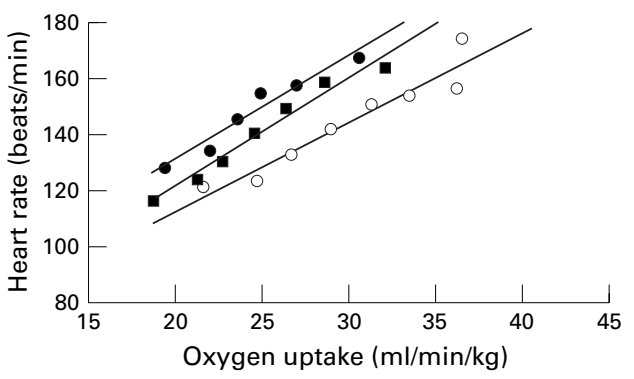

Figure 2 Relation between oxygen uptake and heart rate during graded exercise in patients with transposition of the great arteries and atrial switch (solid circles) and patients with repair of tetralogy of Fallot (solid squares), compared with normal controls (open circles). The data points represent mean values for the different patient groups. 


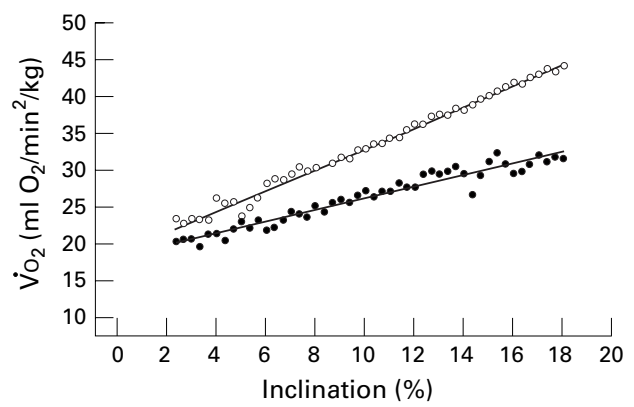

Figure 3 Typical example of the slope of oxygen uptake $v$ exercise intensity during graded treadmill exercise in a 14 year old normal control (open circles) and in a 16 year old patient after repair for tetralogy of Fallot (solid circles). The data represent average values of oxygen uptake in 10 second intervals. Data collected during the first minute of exercise were omitted because of breathing irregularity at onset of exercise.

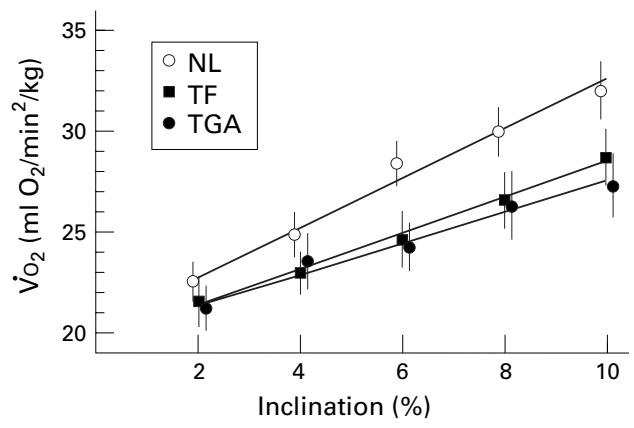

Figure 4 Slopes of oxygen uptake $v$ exercise intensity (time) during graded treadmill exercise for normal controls (NL; open circles), patients after tetralogy of Fallot repair (TF; solid squares), and patients with transposition of the great arteries and atrial switch (TGA; solid circles). The slopes represent the mean value for each group compared for subsequent levels of exercise (inclination of the treadmill). Data collected during the first minute of exercise were omitted because of breathing irregularity at the onset of exercise. The data points represent average values and $95 \%$ confidence intervals of the mean for oxygen uptake $(\mathrm{ml} \mathrm{O} / \mathrm{min} / \mathrm{kg})$.

ANALYSIS OF THE SLOPE OF OXYGEN UPTAKE VERSUS EXERCISE INTENSITY

The adequacy of the oxygen transport system was evaluated by calculating the slope of the increase in oxygen uptake versus exercise intensity (fig 3). Figure 4 gives the mean slope of oxygen uptake versus exercise intensity for the two patient groups compared with the normal controls, for a same number of exercise levels. The slope for oxygen uptake versus exercise intensity averaged $1.77(0.75) \mathrm{ml} \mathrm{O} / \mathrm{min}^{2} / \mathrm{kg}$ in patients with $\mathrm{TF}$ and $1.50(0.64) \mathrm{ml} \mathrm{O}_{2} / \mathrm{min}^{2} / \mathrm{kg}$ in patients with TGA; these values were lower $(\mathrm{p}<0.001)$ than that obtained in the controls $\left(2.42(0.68) \mathrm{ml} \mathrm{O}_{2} / \mathrm{min}^{2} / \mathrm{kg}\right.$ ) (table 1).
The lowest value for the slope of oxygen uptake versus exercise intensity was found in patients with TGA, but the difference between TGA and TF was not significant. The decreased oxygen uptake slope was associated with significantly lower values for the ventilatory anaerobic threshold, expressed as per cent of the normal mean value obtained in children or adolescents of the same age and sex. In patients after total repair for TF, it averaged 85.1 (10.6)\% of normal, and in patients with TGA, 78.0 (13.3)\% (table 1).

To account for the influence of differences in body weight on the magnitude of the work rate increments during graded treadmill exercise, an estimate of the external work rate was calculated for a standard inclination and speed of the treadmill (10\% elevation; speed $5.6 \mathrm{~km} /$ $\mathrm{h})$. In the normal subjects external work averaged $57.2(16.4) \mathrm{W}$, in the patients with TGA it averaged $51.8(17.1) \mathrm{W}$, and in the patients with TF repair, 64 (24.5) W. No significant difference was found between the three groups $(\mathrm{p}>0.05)$.

Above the ventilatory anaerobic threshold, a disproportionate rise in carbon dioxide output against oxygen uptake was found. The steepness of the slope of carbon dioxide versus oxygen uptake was significantly greater in both the patient groups than in the normal controls. The highest value was found in the patients with TGA (table 1). Moreover, in both patient groups, the slope of ventilation versus carbon dioxide output was also greater $(p=0.001)$ than in the normal controls $(41.5$ (13.7) in TF repair and 47.0 (15.4) in TGA, $v 30.3$ (8.5) in the controls).

CLINICAL APPLICATION OF THE SLOPE OF OXYGEN UPTAKE VERSUS EXERCISE INTENSITY

To assess the clinical applications of this concept, we correlated the results of the slope of oxygen uptake against exercise intensity with the clinical outcome after surgery in both patient groups. In 10 of the patients with TF repair, a subnormal value was found $(<1.8$; that is, below the lower limit of the $95 \%$ confidence interval of 1.8 to 3.04 , determined from the data obtained in the 24 normal controls). In the normal subjects no significant correlation was found between the slope of oxygen uptake versus exercise intensity and age $(r=0.093, \mathrm{p}=0.66)$.

Table 1 Slopes of oxygen uptake versus exercise intensity, carbon dioxide output versus oxygen uptake, and ventilation versus carbon dioxide together with anaerobic threshold in patients with congenital heart disease and normal controls

\begin{tabular}{|c|c|c|c|c|c|}
\hline & $\begin{array}{l}\text { Slope } \dot{\mathrm{V}} \mathrm{O}_{2} v \\
\text { exercise intensity } \\
\left(\mathrm{ml} / \mathrm{min}^{2} / \mathrm{kg}\right)\end{array}$ & $\begin{array}{l}\text { Ventilatory } \\
\text { anaerobic threshold } \\
(\mathrm{ml} / \mathrm{min} / \mathrm{kg})\end{array}$ & $\begin{array}{l}\text { Ventilatory } \\
\text { anaerobic threshold } \\
\text { (\% normal) }\end{array}$ & Slope $\dot{V}_{\mathrm{CO}_{2}} v \dot{V}_{\mathrm{O}_{2}}$ & Slope $\dot{V}_{E} v \dot{V}_{C_{2}}$ \\
\hline Normal controls $(n=24)$ & $2.42(0.68)$ & $29.0(3.1)$ & - & $1.05(0.13)$ & $30.3(8.5)$ \\
\hline $\mathrm{TF}(\mathrm{n}=30)$ & $1.68(0.75)^{\star}$ & $24.9(3.6)^{\star}$ & $85.1(10.6)^{\star}$ & $1.20(0.18)^{\star}$ & $41.5(13.7)^{\star}$ \\
\hline TGA $(n=29)$ & $1.50(0.64)^{\star}$ & $22.6(4.7)^{\star}$ & $78.0(13.3)^{\star}$ & $1.26(0.20)^{\star}$ & $47.0(15.4)^{\star}$ \\
\hline
\end{tabular}

Data are means (SD).

${ }^{\star} \mathrm{p}<0.005$, normal controls $v$ patients (TF and TGA).

Slope $\dot{\mathrm{V}}_{2} v \dot{\mathrm{V}}_{2}$, slope of carbon dioxide output $v$ oxygen uptake from oxygen uptake at ventilatory anaerobic threshold until oxygen uptake at heart rate of 170 beats/min; slope $\dot{\mathrm{V}} v \dot{\mathrm{V}} \mathrm{CO}_{2}$, slope of ventilation $v$ carbon dioxide output during graded exercise; $\mathrm{TF}$, tetralogy of Fallot after repair; TGA, transposition of the great arteries after atrial switch operation; $\mathrm{VCO}_{2}$, carbon dioxide output; $\dot{\mathrm{V}}_{2}$, oxygen uptake. Ventilatory anaerobic threshold (\% of normal) is expressed as a percentage of the mean value obtained in normal children and adolescents of same age and sex. 
In the 10 patients after $T F$ repair who had a subnormal value for the slope, seven had moderate to severe pulmonary regurgitation assessed by echocardiographic examination, two had right ventricular dysfunction, and one had an absent left pulmonary artery. In the $20 \mathrm{TF}$ patients with a normal value for this slope (>1.8), only three had a moderate degree of pulmonary regurgitation, while the rest had only trivial regurgitation during echocardiography or on clinical examination.

In all the patients with the atrial switch operation for TGA, we found a dilated and hypocontractile right ventricle during echocardiographic examination, as is usually observed in such patients. Subnormal values for the slope of oxygen uptake versus exercise intensity were found in 17 patients with residual haemodynamic defects such as subvalvar pulmonary stenosis, moderate to severe tricuspid regurgitation, and cardiomegaly on chest $x$ ray. In the other 12 patients with a normal slope of oxygen uptake versus exercise intensity ( $>1.8$ ), no significant residual haemodynamic lesions were observed.

\section{Discussion}

The major finding in this study was the smaller increase of the oxygen uptake versus exercise intensity relation with increasing inclination of the treadmill in the patients with surgically treated congenital heart disease compared with normal controls. This was associated with a subnormal value for the ventilatory anaerobic threshold and a significantly steeper value for the slope of carbon dioxide output against oxygen uptake above the anaerobic threshold. In addition, the slope of ventilation versus carbon dioxide output was higher in both patient groups compared with the normal controls. This is in agreement with previous studies in patients with acquired cardiovascular disorders (mostly ischaemic heart disease and chronic heart failure), where significantly lower values for the slope of oxygen uptake against work rate were found, ${ }^{7911}$ and a steeper value of the slope of carbon dioxide output versus oxygen uptake. ${ }^{5}$ Moreover, in patients with chronic heart failure, ${ }^{22}$ and more specifically after TF repair ${ }^{20}$ or after the Mustard operation for TGA, ${ }^{23}$ an increase in the slope of ventilation against carbon dioxide output has been reported; this has been attributed to maldistribution of pulmonary blood flow, with increased physiological dead space ventilation. In our present study on patients with congenital heart disease, as well as in other studies in patients with ischaemic heart disease, ${ }^{5}$ the reduced slope of oxygen uptake is also associated with a steeper slope of carbon dioxide output versus oxygen uptake, which is thought to reflect the buffering of lactic acid by bicarbonate. $^{82}$ The excessive ventilatory response to carbon dioxide has been ascribed to the use of the respiratory cycle as an auxiliary pump in patients with decreased ventricular function, to improve venous return and consequently pulmonary blood flow. ${ }^{25}$ The reduced slope of oxygen uptake versus exercise intensity, together with the inefficient ventilatory response to carbon dioxide, can be considered as factors limiting exercise capacity as they reflect impaired oxygen delivery to the exercising tissues and wasted ventilation.

In previous studies on children and adolescents with congenital heart disease, aerobic exercise performance has often been assessed by determining maximum oxygen uptake or anaerobic threshold. ${ }^{1026}$ However, both measures can be criticised, especially in the paediatric age group. Determination of maximum oxygen uptake is dependent on motivation, which can be low in children, while calculation of the anaerobic threshold is influenced by several factors that increase the variance of the result, including differences in the exercise protocol, the method used for threshold determination, and interobserver differences. ${ }^{2-4} \mathrm{We}$ studied the clinical value of the slope of oxygen uptake versus exercise intensity in two groups of patients known to have reduced aerobic exercise performance. Subnormal values have been reported for maximum oxygen uptake and ventilatory anaerobic threshold in patients with TGA and atrial switch ${ }^{26}$ and in patients with $\mathrm{TF}$ repair. ${ }^{28}$ In addition, a reduction in oxygen uptake in relation to exercise intensity has also been found in exercise studies examining the kinetics of gas exchange. For example, in children with total repair of TF and in those with an atrial switch operation for TGA, a slowed rate of oxygen uptake was observed during a constant level of exercise. ${ }^{29}$ This may explain the lower value for the slope of oxygen uptake versus exercise intensity that we found in both patient groups in the present study. It also confirms the subnormal value of these determinants of aerobic exercise function, and suggests impaired oxygen delivery to the exercising tissues. ${ }^{30}$

In patients with TGA and atrial switch operation, the reduced oxygen delivery to the exercising tissues has been attributed to a lower, and inappropriate, right (systemic) ventricular ejection fraction and a subnormal cardiac output during moderate exercise, caused by relative obstruction to flow. ${ }^{31}{ }^{32}$ In patients who have had total repair of TF, the lower value of the slope of oxygen uptake versus exercise intensity - together with a steeper value of the slope of carbon dioxide output versus oxygen uptake above the ventilatory anaerobic threshold, and an increased slope of ventilation versus carbon dioxide output - results from haemodynamic dysfunction. This may be caused by pulmonary valve incompetence,,$^{33}$ an impaired ejection fraction, ${ }^{34}$ or impaired chronotropic exercise response, ${ }^{28}{ }^{35}$ resulting in a subnormal cardiac output during submaximal and maximal exercise. ${ }^{35}$ In our present study the lowest values for the slope of oxygen uptake against exercise intensity were found in the patients with residual haemodynamic defects, such as severe pulmonary regurgitation after TF repair and subvalvar pulmonary stenosis after atrial switch operation in TGA. 
COMMENT ON THE METHODS

In most previous studies investigating oxygen uptake during exercise in patients with cardiovascular disease, the slope of oxygen uptake versus work rate on a bicycle ergometer was calculated..$^{7910}$ In the present study we used graded treadmill exercise and assessed the relation between oxygen uptake and exercise time during a one minute incremental exercise test (reflecting exercise intensity). Although external work can be difficult to quantify during treadmill exercise owing to differences in walking pattern, a rough estimate can be made by using standard equations. ${ }^{16}$ As body mass may influence the work rate increment during graded treadmill exercise, it could be hypothesised that differences in body mass between different groups may have influenced the rate of increase in external work performed during the exercise. We calculated the difference in work rate for a given inclination $(10 \%)$ and standard speed $(5.6 \mathrm{~km} / \mathrm{h})$ in the three different groups. This showed that the magnitude of the difference in work rate between patients with TGA and normal controls $(9.6 \%)$ was much smaller than the difference in the slope of oxygen uptake against exercise (38\%). Furthermore, as oxygen uptake was normalised for body weight, this factor was cancelled out. Therefore a lower body mass in patients with TGA cannot explain the lower slope of oxygen uptake against exercise intensity.

In patients with congenital heart disease, one should take into account that the slope of oxygen uptake versus work rate is dependent on the exercise protocol. In a comparison between a one minute incremental exercise protocol and a four minute (steady state) graded exercise protocol in a large series of 540 patients with congenital heart disease, Wesse ${ }^{10}$ reported significantly higher values for the slope of oxygen uptake versus work rate in the four minute test than in the one minute test. Therefore each laboratory should determine its own appropriate normal standards for comparing clinical values.

\section{STUDY LIMITATIONS}

We assessed the results of this new index of cardiovascular exercise function in congenital heart disease in relation to the clinical outcome after surgical correction. Direct measurements of cardiac function using intracardiac pressures and determination of myocardial contractility by analysis of $\mathrm{dP} / \mathrm{dt}$ were not available, neither were measurements of cardiac output or blood pressure response during exercise. However, on the basis of echocardiography and clinical examination we were able to show that the lowest values for the slope of oxygen uptake versus exercise intensity occurred in patients with the most severe pulmonary regurgitation after $\mathrm{TF}$ repair, and in patients with significant residual subvalvar pulmonary stenosis and or cardiomegaly in TGA.

Despite these limitations, this new index appears useful as it can discriminate between patients with more severe haemodynamic sequelae and those with more trivial haemodynamic lesions.
CONCLUSIONS

Our study shows that the determination of the slope of oxygen uptake versus exercise intensity is a reproducible, valid, and comprehensive index of cardiorespiratory exercise function. It provides information on oxygen flow to the exercising tissues, which may be a limiting factor in exercise performance in patients with congenital heart disease. Determination of this slope is particularly useful in patients with congenital heart disease, as a subnormal value is associated with important residual haemodynamic defects both after TF repair and after the atrial switch operation for TGA.

This study was supported in part by grants of the National Fund for Research in Pediatric Cardiology and the National Fund for Medical Research (3.020.94). We gratefully acknowledge the help of D Schepers MS, during the statistical analysis of the data.

1 Myers J, Ashley E. Dangerous curves. A perspective on exercise, lactate, and the anaerobic threshold. Chest 1997;111:787-95.

2 Cabrera ME, Chizeck HJ. On the existence of a lactate threshold during incremental exercise: a systems analysis. $\mathcal{F}$ Appl Physiol 1996;80:1819-28.

3 Shimizu M, Myers J, Buchanan N, et al. The ventilatory threshold: method protocol and evaluator agreement. Am Heart f 1991;122:509-16.

4 Meyer K, Hajric R, Westbrook S, et al. Ventilatory and lactate threshold determinations in healthy normals and cardiac patients: methodological problems. Eur f Appl Physiol 1996;72:387-93.

5 Koike A, Hiroe M, Adachi $\mathrm{H}$, et al. Anaerobic metabolism as an indicator of aerobic function during exercise in cardiac patients. 7 Am Coll Cardiol 1992;20:120-6.

6 Reybrouck T, Mertens L, Kalis N, et al. Dynamics of respiratory gas exchange during exercise after correction of respiratory gas exchange during exercise after correction of

7 Hansen JE, Sue DY, Oren A, et al. Relation of oxygen uptake to work rate in normal men and men with circulatory disto work rate in normal men and men
orders. Am $\mathcal{F}$ Cardiol 1987;59:669-74.

orders. Am f Cardiol 1987;59:669-74.
8 Wasserman, K, Hanssen JE, Sue DY, et al. Principles of exercise testing and interpretation (2nd ed). Philadelphia: Lea and Febiger, 1994

9 Cohen-Solal A, Chabernaud JM, Gourgon R. Comparison of oxygen uptake during bicycle exercise in patients with chronic heart failure and normal subjects. $f$ Am Coll Cardiol 1990;16:80-5.

10 Wessel HU. Integrated cardiopulmonary approach to exercise testing in pediatrics. Pediatrician 1986;13:26-33.

1 Myers J, Buchanan N, Walsh D, et al. Comparison of the ramp versus standard exercise protocols. $f$ Am Coll Cardiol 1991;17:1334-42.

12 Tanner JM, Whitehouse RH. Clinical longitudinal standards for height, weight, height velocity and weight velocity, and stages of puberty. Arch Dis Child 1976;51:170-9.

13 Reybrouck T, Deroost F, Van der Hauwaert LG. Evaluation of breath-by-breath measurement of respiratory gas exchange in pediatric exercise testing. Chest 1992;102:14752.

14 Washington RL, Bricker JT, Alper BS, et al. Guidelines for exercise testing in the pediatric age group. From the committee on atherosclerosis and hypertension in children, council on cardiovascular disease in the young, the American Heart Association. Circulation 1994;90:2166-79.

15 Hughson RL, Northey DR, Xing HC, et al. Alignment of ventilation and gas fraction for breath-by-breath respiratory gas exchange calculations in exercise. Comput Biomed Res 1991;24:118-28.

16 Adams GM. Exercise physiology. Laboratory manual, $2^{\text {nd }}$ ed. Madison (Wisconsin): Brown and Benchmark, 1994:80-1.

17 Beaver WL, Wasserman K, Whipp BJ. A new method for detecting anaerobic threshold by gas exchange. $f$ Appl Physiol 1986;60:2020-7.

18 Reybrouck T, Weymans $M$, Stijns $\mathrm{H}$, et al. Ventilatory anaerobic threshold in children: age and sex differences. anaerobic threshold in children: age
Eur F Appl Physiol 1986;54:278-84.

19 Wasserman K, Zhang YY, Gitt A, et al. Lung function and exercise gas exchange in chronic heart failure. Circulation 1997;96:2221-7.

20 Clark AL, Gatzoulis MA, Redington AN. Ventilatory responses to exercise in adults after repair of tetralogy of Fallot. Br Heart F 1995;73:445-9.

21 Bland MJ, Altman DH. Statistical methods for assessing agreement between two methods of clinical measurement. Lancet 1986;i:307-10.

22 Chua TP, Clark AL, Amadi AA, et al. Relation between chemosensitivity and the ventilatory response to exercise in chronic heart failure. $\mathcal{F}$ Am Coll Cardiol 1996;27:650-7.

23 Gilljam T, Eriksson B, Sixt R. Cardiac output and pulmonary gas exchange at maximal exercise after atrial redirection for complete transposition. Eur Heart $\mathcal{f}$ 1998:19:1856-64. 
24 Wasserman K, Beaver WL, Whipp BJ. Gas exchange threshold and the lactic acidosis (anaerobic) threshold. Circulation 1990;81(suppl 11):14-30.

25 Janicki JS, Sheriff DD, Robotham JL, et al. Cardiac output during exercise: contributions of the cardiac, circulatory, and respiratory systems. In: Rowell LB, Shepherd JT, ed Handbook of physiology. Section 12. Exercise: regulation and integration of multiple systems. Oxford: Oxford University Press, 1996:649-704

26 Douard H, Labbé L, Barat JL, et al. Cardiorespiratory response to exercise after venous switch operation for transposition of the great arteries. Chest 1997;111:23-9

27 Reybrouck T, Gewillig M, Dumoulin M, et al. Cardiorespiratory exercise performance after Senning operation for transposition of the great arteries. Br Heart $\mathscr{f}$ 1993;70:175-9.

28 Reybrouck T, Weymans $M$, Stiins $H$, et al. Exercise testing after correction of tetralogy of Fallot: the fallacy of a reduced heart rate response. Am Heart $\mathcal{f}$ 1986;112:998-1003.

29 Mocellin R, Gildein P. Velocity of oxygen uptake response at the onset of exercise: a comparison between children after cardiac surgery and healthy boys. Pediatr Cardiol 1999;20: $17-20$.
30 Wasserman K. Diagnosing cardiovascular and lung pathophysiology from exercise gas exchange. Chest 1997;112: physiology

31 Page E, Perrault H, Flore P, et al. Cardiac output response during dynamic exercise after atrial switch repair for transposition of the great arteries. Am F Cardiol 1996;77:892-5.

32 Gewillig M, Balaji S, Mertens B, et al. Risk factors for arrhythmia and death after Mustard operation for simple transposition of the great arteries. Circulation 1991;84:18792.

33 Rowe SA, Zakha KG, Manolo TA, et al. Lung functions and pulmonary regurgitations limit exercise capacity in postoperative tetralogy of Fallot. F Am Coll Cardiol 1991;17:461-6.

34 Vetter HD, Reichaert B, Seidel P, et al. Non-invasive assessment of right and left ventricular volumes 11 to 24 years after corrective surgery on patients with tetralogy of Fallot. Eur 7 Cardiothorac Surg 1990;4:24-8.

35 Perrault H, Drblik SP, Montigny M, et al. Comparison of Perrault $\mathrm{H}$, Drblik SP, Montigny $\mathrm{M}$, et al. Comparison of 15 years of adjustments to exercise in adolescents 8 to 15 years of age after correction of tetralogy of Fallot, ventricular septal defect or atrial septal defect. Am f Cardio

\section{IMAGES IN CARDIOLOGY}

\section{Visualising fatty deposits in familial arrhythmogenic right ventricular cardiomyopathy by magnetic resonance imaging}

This 70 year old man had familial arrhythmogenic right ventricular cardiomyopathy (ARVC) with recurrent ventricular tachycardia since the age of 41 . The diagnosis of ARVC was made on the basis of recurrent, refractory ventricular tachycardia, echocardiographic findings, a positive family history, and the detection on magnetic resonance imaging (MRI) of fatty deposits in the right ventricle. Transoesophageal echocardiography (top) showed a dilated right ventricle and focal thinning of the right ventricular wall apicoseptally and apicolaterally with two localised bulges in the right ventricular wall (arrows) (RV, right ventricle; LV, left ventricle). MRI (bottom) showed an almost akinetic thin wall of the right ventricle with fatty deposits, confirming the diagnosis of familial ARVC. Fatty deposits are seen in the atrioventricular groove (1); the right ventricular free wall is completely replaced by fatty tissue (2), which also extends to the apex and part of the lateral wall of the left ventricle (3); pericardium is seen at the base of the left and right ventricles (4); slow flow artefacts are particularly predominant in the right atrium (5).

MRI is a non-invasive method for detecting isolated fatty deposits in the myocardium of the right ventricle. The abnormalities shown by MRI are generally the starting point for the observed ventricular tachycardias. The differential diagnosis has to take account of Uhl's anomaly, which also involves localised involvement of the right ventricle. In contrast with ARVC, wall areas in Uhl's anomaly are characterised by a complete absence of
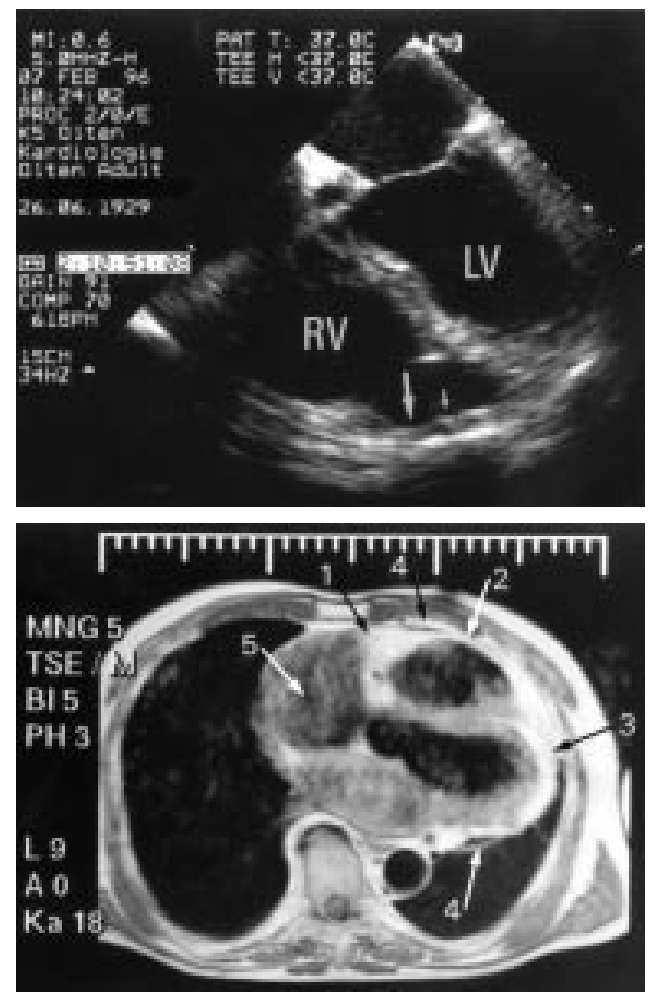

myocardial cells. In such wall sections the endothelium and epithelium are in direct apposition.

FRANZ F IMMER MICHEL ROMANENS HUGO SANER 